\title{
COMPARISON OF THE EFFECTIVENESS OF STEREOTACTIC RADIOSURGERY FOR SPINAL OLIGOMETASTATIC DISEASE WITH SURGICAL AND CONVENTIONAL EXTERNAL BEAM RADIOTHERAPY DETERMINED BY LOCAL CONTROL, OVERALL SURVIVAL, PAIN RESPONSE AND TOXICITIES: A SYSTEMATIC REVIEW.
}

\author{
Priyanshu Saha*, Thamer Hamdan", Bisola Ajayi" ${ }^{* *}$ Cristina Lupu\%, \\ Timothy Bishop ${ }^{\&}$, Jason Bernard ${ }^{\wedge} \&$ Darren Lui ${ }^{\circledR}$, \\ *MB,BS Student, Saint George's University of London. "FRCS, FRCP, FACS, FICS, American Board \\ (Neuro Ortho) Professor of Orthopaedic Surgery, Research fellow, Saint George's University. ${ }^{* *}$ PA-R \\ Ortho/Spine Physician Associate. ${ }^{\circ}$ Spine CNS ${ }^{\&}$ FRCS. $M$ MD, FRCS. ${ }^{\circledR}$ FRCS, Saint George's University, \\ London, UK.
}

\begin{abstract}
The aim of this study is to determine whether there is a benefit of using stereotactic radiosurgery (SRS) versus surgery or conventional external beam radiotherapy (cEBRT) for spinal oligometastatic disease in terms of tumor control, pain control, toxicity and morbidity.

This systematic review was conducted by searching electronic databases such as Pubmed, Medline (Ovid) and Oxford Academic using an adapted version of the Preferred Reporting Items for Systematic Reviews and Meta-Analyses" (PRISMA) checklist. Retrospective and prospective studies were identified and investigated for methods of treatment such as SRS, surgery and radiotherapy of spinal oligometastatic disease. Local Control rates, Overall Survival (OS) rates, Pain Response (PR) and toxicities were extracted to be investigated and compared. A study eligibility criterion was made to ensure that results were valid, statistically significant and relevant to the investigation.

One hundred and five articles were relevant to oligometastatic disease with the mention of spinal metastases however 89 of these articles were excluded based on irrelevance to investigation, title, abstract and duplication. Sixteen full text articles were thoroughly screened including 9 of them in the review. For 601 patients the average one year local control rate was $89 \%$, average one year overall survival rate was $88 \%$, evidence of pain relief was present, and some patients suffered low grade toxicities.

In conclusion, stereotactic radiosurgery is an excellent modality of treatment of spinal oligometastatic disease as local control rates, overall survival rates, pain response and toxicities indicate improved outcomes in comparison to studies that investigated the same factors with the treatment of surgery or cEBRT. However, there is a lack of data here to make statistically valid claims and require more studies \& data to be analyzed and compared.

Key words: stereotactic radiosurgery, surgery, conventional external beam radiotherapy, spinal oligometastatic disease
\end{abstract}

\section{Introduction} ligometastatic disease was termed by
Hellman and Weichselbaum in the Journal of Clinical Oncology 1995. It describes as a state of cancer with limited number of detectable intermediary metastatic tumors with limited disease burden between localized lesions and widespread metastasis ${ }^{1}$. The definition is unclear as there is no consensus as to how many lesions or metastasis is associated with the oligometastatic state as it can depend on the nature of the primary tumor and the time interval between diagnosis between the primary tumor and oligometastatic disease.

Several definitions have been proposed to describe this metastatic state depending on the primary tumor, the number of 
lesions, the natural history of the disease and previous treatment of the disease $\mathrm{e}^{2}$; Oligorecurrence: Limited metastases in the presence of a treated (controlled) primary lesion. Sync-oligometastasis: $\leq 5$ metastatic or recurrent lesions in the presence of untreated (uncontrolled) primary tumor. Synchronous oligometastasis: Oligometastatic disease is detected at the time of diagnosis of the primary tumor; therefore there is an untreated (uncontrolled) primary tumor. Metachronous oligometastasis: Development of oligometastatic disease after treatment of the primary tumor; interval for classification of metachronous versus synchronous is not standardized; between controlled primary lesion except for concomitant primary and distant recurrence.

Oligometastatic lesions can be detected early by imaging techniques such as Magnetic Resonance Imaging (MRI) or positron emission tomography (PET). These new advancements in imaging have created opportunities for early detection and prevention of further metastasis, myelopathy and radiculopathy which ultimately improves mortality and life quality. An example of MRI/bone scan of oligometastatic disease of spine is shown in figure 1.

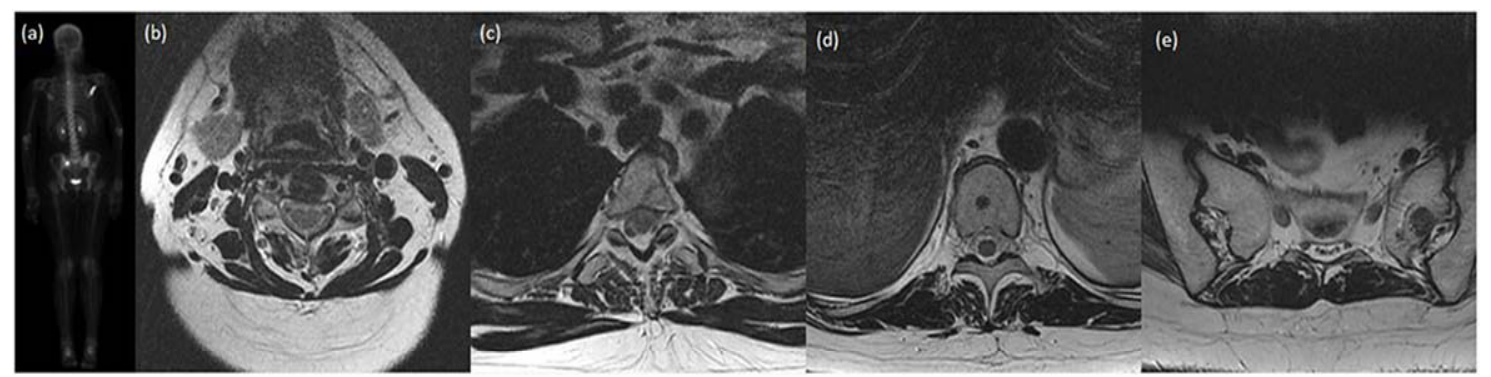

Figure 1: KL Zeng et al 2019³ : MRI/bone scan of lady with oligometastatic disease (a) sites of metastases, (b) $\mathrm{C} 4$ marrow replacement, (c) posterior $\mathrm{T} 3$ body, (d) around $\mathrm{T} 10$ vertebral body, (e) $13 \mathrm{~mm}$ lesion on left sacral ala.

The spine is a common site of secondary metastasis. Approximately $70 \%$ of bony metastases are in the spinal vertebral bodies. In the USA and UK, respectively every year there are 20,000 and 4000 new cases of spinal cord compression due to spinal metastases. There are three main modalities of treatment which are radiotherapy, surgery and radiosurgery. In addition, systemic therapy such as chemotherapy, radionuclide therapy, immunotherapy and hormonal therapy can also be used to treat specific metastases. All these modalities are not isolated forms of treatment but can give as adjuvant treatment to other modalities depending on the type of cancer.
Historically the aim of treating spinal compression has been to treat the patient palliatively with radiotherapy or surgery to achieve pain relief, tumor control and neurological function preservation. The neurological, oncologic, mechanical instability, and systemic disease of the patient (NOMS) framework was developed by Mark Bilsky (Figure 2) which considers four aspects of oncologic status: tumor histology, chemosensitivity \& hormonal sensitivity, and radiosensitivity to assess the status of the spinal metastases. This framework is used to decide which treatment modality is the most appropriate. 


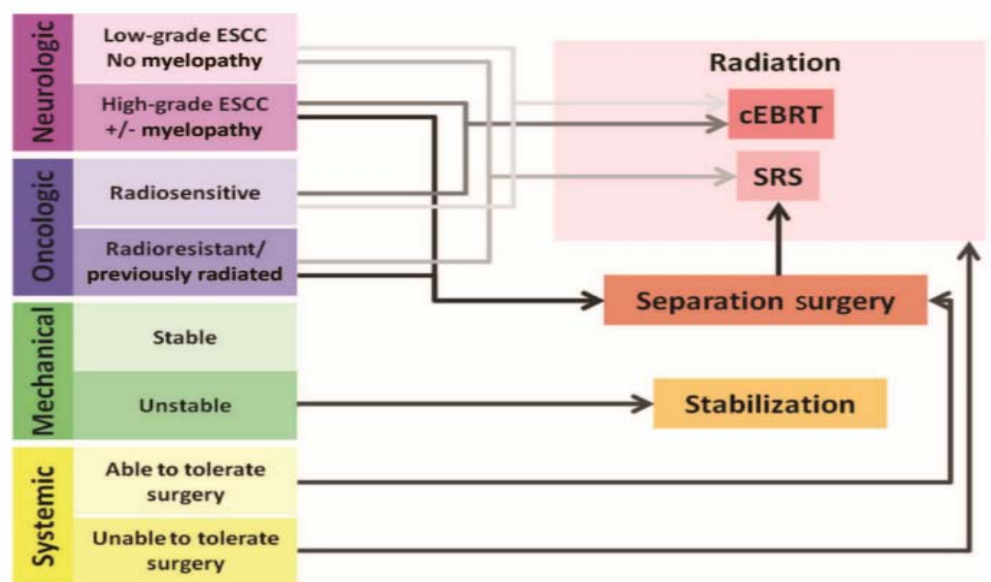

Figure 2: Laufer et al., $\mathbf{2 0 1 3}^{4}$, Schematic diagram of the NOMS framework developed, abbreviations: cEBRT - conventional external beam radiotherapy, SRS - Stereotactic Radiosurgery

The neurologic assessment grades epidural spinal cord compression (ESCC) and the clinical assessment of myelopathy and/or functional radiculopathy. ESCC is a 6 point grading system as illustrated in figure 3. The $0-1 \mathrm{~b}$ are low ESCC graded and considered for radiation as initial treatment. Grade 2-3 are high ESCC graded due to spinal cord compression and requires surgical decompression. Oligometastatic disease is low graded
ESCC of 0 or 1 due to its limited tumor size containment within the vertebral body. As it does not affect the cord, myelopathy and radiculopathy are not expected. Furthermore, mechanical stability is not affected. Hence following the NOMS framework, SRS or cEBRT is the preferred line of treatment unless tumor histology proves to be radioresistant/previously radiated.

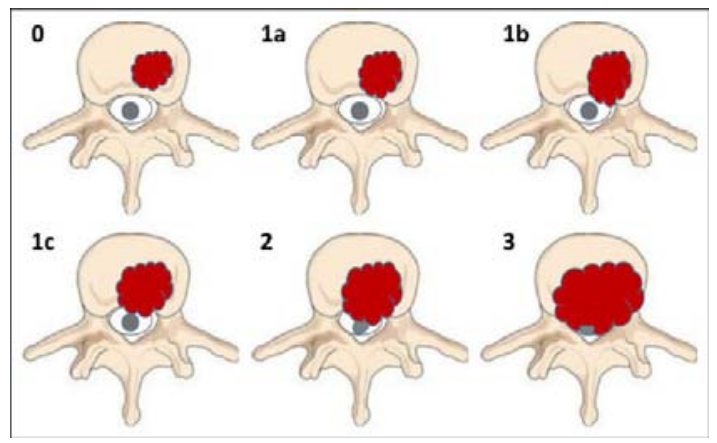

Figure 3: Tseng $2017^{5}$, Schematic of the 6-point epidural spinal cord compression (ESCC) grading scale proposed by Bilsky et al. "Grade 0 denotes bone-only disease; 1a, epidural impingement, without deformation of the thecal sac; $1 \mathrm{~b}$, deformation of the thecal sac, without spinal cord abutment; 1c, deformation of the thecal sac with spinal cord abutment, but without cord compression; 2, spinal cord compression, but with CSF visible around the cord; and 3, spinal cord compression, no CSF visible around the cord"

Radiotherapy has been used on bone metastasis for palliative purposes throughout the 20th century. Radiotherapy is delivered by 1 or 2 beams of radiation without high precision and highly conformal treatment techniques. Conventional external beam radiotherapy (cEBRT) is most often the initial form of treatment, the limitation is that it lacks high precision hence nearby healthy tissue is damaged. The spinal cord is intolerant to radiation this can be quite damaging and cause impairment to neurological elements ${ }^{6}$. 
Historically when radiation was not viable, surgery was undertaken to decompress the spine by laminectomy or vertebral body resection followed by stabilization ${ }^{6}$. Advancements in minimally invasive techniques such vertebroplasty and kyphoplasty have been proven to minimalize tissue damage, obtaining better surgical outcome, lowering morbidity and improving life quality $^{6}$. Radical en bloc spondylectomy is the newer method of surgery to completely ablate the oligometastatic tumors however due to the open nature of surgery, high morbidity and complication rates were expected as the patients are already weakened by their underlying metastatic disease ${ }^{7}$. The Patchell trial 2005 compared the effect of radiotherapy and surgery of spinal metastatic disease ${ }^{8}$. However, it was not applicable to oligometastatic disease as its results are based off wider metastasis that causes loss of ambulation and compared results by overall ambulation, maintenance of ambulation, recovery of ambulation, bladder and bowel incontinence and survival. As these are extreme symptoms that are not expected from oligometastasis it can be difficult to interpret the findings of those results. However, the findings of the randomized trial can be used to compare the outcomes of surgical and radiotherapy treatment and radiotherapy alone. When comparing surgery and radiotherapy vs radiotherapy alone it was found: that overall ambulation was $84 \%$ vs $57 \%$, maintenance of ambulation was $94 \%$ vs $74 \%$, recovery of ambulation was $62 \%$ vs $19 \%$, bladder and bowel continence was 155 vs 17 days and overall survival was 122 days to 100 days $^{6}$. This trial indicated that surgical and radiotherapy outcome was superior to radiotherapy alone and can be considered to as the better treatment for oligometastasis as well. However, it is difficult to apply it directly to the oligometastatic state as ambulation is not affected and surgical management could cause more morbidity than benefit.

Ultimately, the optimal primary treatment or adjuvant therapy for spinal metastasis was shifted to spinal radiosurgery which is an advancement of cEBRT. It is a development from cEBRT as it has better tumor control and pain control. Furthermore, it has lower morbidity than open surgery. Barzilai et $\mathrm{al}^{9}$. investigated the comparison between surgery with or without RT versus cEBRT versus SSRS on the treatment of oligometastatic disease of the spine. He compared the 1-year Local Control rate of each of the treatments. It was found that surgery with or without RT $(\mathrm{n}=129)$ had $65.5 \%$ 1-year local control, cEBRT $(n=23)$ had $57.1 \%$ 1-year local control rate and SRS $(\mathrm{n}=63)$ had $84.8 \% 1$ year local control rate [9]. The Kaplan Meier Curves further supporting this evidence can be found in Appendix A. This study provides evidence to support that SRS is the best treatment for treating this state of cancer. However, it important to consider that the sample sizes of each of the treatment groups are unequal and the difference in local control rate can be due to factors such as difference in surgical skill and approach hence could affect the validity of the investigation. Stereotactic Radiosurgery (SRS), Stereotactic Body Radiotherapy (SBRT) or Stereotactic ablative body radiotherapy (SABR) are assumed to be synonymous terms for treatment using the Gammaknife or the Cyberknife. SRS is a linear accelerator mounted on a robotic arm capable of firing several $\mathrm{x}$-ray/gamma/proton beams of radiation in any desired angle whilst having the patient immobilized, shown in figure 4. It is a method of delivering dosage of radiation therapy in a single or limited number of fractions, with a high degree of conformity allowing treatment near spinal cord and other critical structures, to completely ablate the tumor. For the purpose of this systematic review 
focusing on the spinal oligometastasis, the term Spinal Stereotactic Radiosurgery (SSRS) will be consistently used to describe radiosurgery treatment, as SBRT AND SABR can indicate treatment for metastatic tumors of other organs.

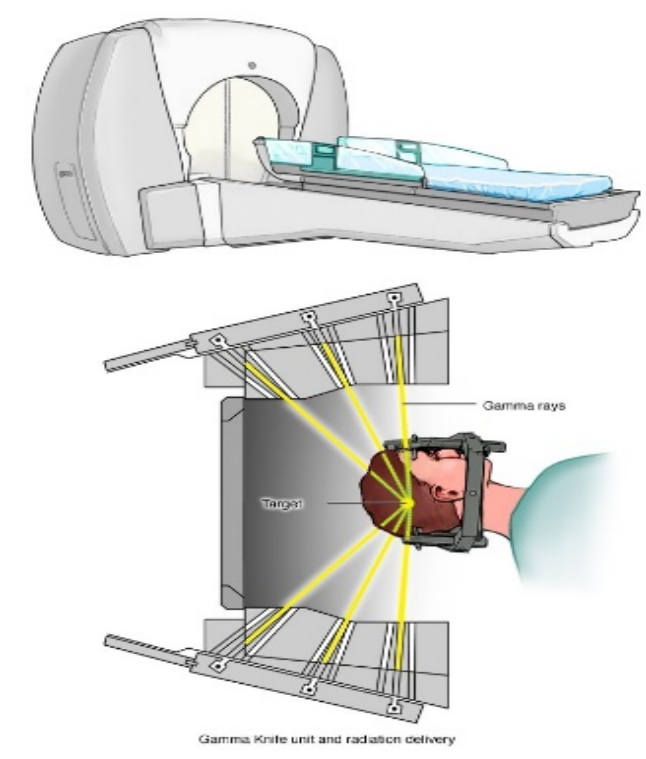

Figure 4. Mayo Clinic 2019 - Schematic of Gammaknife machine ${ }^{10}$.

\section{Methods}

The systematic review was written by in guidance of an adapted version of the Preferred Reporting Items for Systematic Reviews and Meta-Analyses (PRISMA) checklist $^{11}$. An electronic database search was conducted on PubMed, Medline (Ovid) and Oxford Academic electronic databases spanning from October 2019January 2020.

Articles investigating SSRS/SBRT, surgery and radiotherapy treatment on spinal oligometastasis were screened thoroughly if those terms were mentioned in the title or abstract, furthermore, results including local control, overall survival, pain response and toxicities in the abstract was important for screening as well. As it was quite difficult to find studies on spinal oligometastasis specifically, more general terms such as bone metastases was used to find more information. All articles after 1995 were considered, as research on this topic is limited. Reference studies found on articles that were screened initially were also accessed and assessed for further data inclusion. All studies were published in English and had been published on different journals or clinical articles. Some reported either or all results of overall survival, local control, pain response and toxicities after treatment of SSRS using different dosage fractionation. The populations investigated underwent SSRS to treat oligometastatic disease with or without prior radiotherapy or surgery.

Local control, overall survival, pain response and toxicities were the main extrapolations from the studies for comparison. Furthermore, dosage fractionation of SSRS was recorded to compare if there was an effect of dosage on the outcomes. Toxicities were also recorded to investigate qualitative outcomes and comparison to morbidities of surgical treatment. Tumor histology was excluded as some studies mentioned it and some didn't, furthermore it did not show any difference in outcome of treatment. Some studies included comparison with polymetastatic disease of spine and compared outcomes, this was excluded when analyzing Kaplan Meier Curves or data tables. Only 
statistically significant data was chosen to make valid comparisons between Local Control or Overall Survival rates ${ }^{7-18}$.

\section{Results}

Nine studies were investigated that had complete relevance to the chosen topic. Although all the studies didn't have all preferred factors for investigation, all of them gave local control rates that could be used for comparison with supporting data of overall survival rates, pain response and toxicities. Table I shows the summary of patient population, dose fractionation, follow up time, local control, overall survival rate, pain response and toxicities of included investigated studies. Other data are shown in tables II-III and graphs 1-3.

Table I. Summarization of patient population, dose fractionation, follow up time, local control, overall survival rate, pain response and toxicities of included investigated studies

\begin{tabular}{|l|l|l|l|l|l|l|}
\hline Study & $\begin{array}{l}\text { Patient } \\
\text { Population }\end{array}$ & $\begin{array}{l}\text { Dose } \\
\text { fractionation } \\
\text { Gy/fraction }\end{array}$ & $\begin{array}{l}\text { Follow up } \\
\text { after SSRS }\end{array}$ & $\begin{array}{l}\text { Local } \\
\text { Control } \\
\text { (LC) }\end{array}$ & $\begin{array}{l}\text { Overall } \\
\text { Survival } \\
\text { rate (OS) }\end{array}$ & $\begin{array}{l}\text { Pain Response } \\
\text { (PR) }\end{array}$ \\
\hline Ho et al ${ }^{7}$ & n=209 & $\mathbf{2 4 / 1 , 1 8 / 1}$ & 12 months & $\mathbf{8 5 \%}$ & $\mathbf{9 5 \%}$ & NR \\
2016 & & $16 / 1,27 / 3$ & 24 months & $\mathbf{8 2 \%}$ & $\mathbf{8 4 \%}$ & $\mathbf{6 0 \%}$ \\
\hline
\end{tabular}

Toxicities: $11 \%$ experienced grade $2 / 3 / 4$ GI toxicity, $3 \%$ experience grade 3 myelopathy $16 \%$ developed vertebral compression fractures

\begin{tabular}{l|l|l|l|l|l|l|}
\hline $\begin{array}{l}\text { Chang et } \\
\text { al }{ }^{12} 2017\end{array}$ & $\mathrm{n}=60$ & $20 / 1,24 / 2$ & 12 months & $92 \%$ & $90 \%$ & NR \\
\hline \multicolumn{7}{|l|}{ Toxicities: $29 \%$ experience grade $1 / 2 / 3$ toxicities including nausea, fatigue, pain flare, esoph } \\
\hline
\end{tabular}

Toxicities: $29 \%$ experience grade $1 / 2 / 3$ toxicities including nausea, fatigue, pain flare, esophagitis, transient radiculitis, diarrhea and vertebral compression fracture

\begin{tabular}{|l|l|l|l|l|l|l|}
\hline $\begin{array}{l}\text { Chaw } \\
2017\end{array}$ & $\mathrm{n}=45$ & $21-30 / 3$ & 14 months & $83 \%$ & 95.7 & $\begin{array}{l}10 / 12(83 \%) \\
\text { reported back } \\
\text { pain prior to } \\
\text { SSRS report } \\
\text { pain relief }\end{array}$ \\
\hline
\end{tabular}

Toxicities: $47 \%$ experienced grade $1 / 2$ acute toxicities including nausea, fatigue, diarrhea and dysphagia

\begin{tabular}{|l|l|l|l|l|l|}
\hline $\begin{array}{l}\text { Thibault et } \\
\text { al }{ }^{14} 2014\end{array}$ & $n=13$ & 12 months & $83 \%$ & $64 \%$ & NR \\
\hline \multicolumn{7}{|l|}{ Toxicities: 23\% Vertebral compression fractures } \\
\hline
\end{tabular}

\begin{tabular}{|c|c|c|c|c|c|c|}
\hline $\begin{array}{l}\text { Silva et } \text { al }^{15} \\
2018\end{array}$ & $n=25$ & $\begin{array}{l}27 / 3 \\
35 / 5\end{array}$ & 12 months & $100 \%$ & NR & $\begin{array}{l}\text { Improvement in } \\
\text { pain }\end{array}$ \\
\hline
\end{tabular}

\begin{tabular}{l|l|l|l|}
2018 & $35 / 5$ & \\
\hline Toxiticities: grade 1/2 fatigue, GI mucosal, skin
\end{tabular}

\begin{tabular}{|l|l|l|l|l|l|l|}
\hline $\begin{array}{l}\text { Barzilai et } \\
\text { al }^{9} 2019\end{array}$ & $\mathrm{~N}=63$ & $\mathbf{2 4 / 2}$ & $\mathbf{8 4 . 8 \%}$ & $\begin{array}{l}\text { Not } \\
\text { statistically } \\
\text { significant }\end{array}$ & $\begin{array}{l}\text { Drastic } \\
\text { improvement in } \\
\text { pain }\end{array}$ \\
\hline
\end{tabular}

\section{Toxiticities: NR}

\begin{tabular}{|l|l|l|l|l|l|l|}
\hline $\begin{array}{l}\text { Yamada et } \\
\text { al }^{16} \mathbf{2 0 0 8}\end{array}$ & $\mathrm{n}=103$ & $\mathbf{1 8 - 2 4} / 1$ & $\mathbf{2 4}$ months & $\mathbf{9 3 \%}$ & NR & NR \\
\hline
\end{tabular}

Toxiticities: NR

\begin{tabular}{|c|c|c|c|c|c|c|}
\hline $\begin{array}{l}\text { Ahmed et } \\
\text { al }^{17} 2012\end{array}$ & $n=63$ & $24 / 3$ & 12 months & $\begin{array}{l}92.1 \% \\
\text { without } \\
\text { previous } \\
\text { RT, } 83.3 \% \\
\text { with } \\
\text { previous } \\
\text { RT }\end{array}$ & $52.2 \%$ & $\begin{array}{l}\text { Increases in pain } \\
\text { reported }\end{array}$ \\
\hline
\end{tabular}

Toxiticities: Grade 1/2/3 toxicities were present

\begin{tabular}{|l|l|l|l|l|l|l|}
\hline $\begin{array}{l}\text { Kam et al } \\
2019\end{array}$ & $\mathbf{n}=20$ & $\begin{array}{l}35 / 5 \\
50 / 5\end{array}$ & 12 & $91 . \%$ & $79.6 \%$ & $\begin{array}{l}\text { 66\% had 1 year } \\
\text { pain control } \\
\text { with absence of } \\
\text { pain of no use of } \\
\text { analgesics after } \\
\text { SSRS }\end{array}$ \\
& & & & & & \\
\hline
\end{tabular}


Table II: Local Control rates of Radiosurgery Treatment of spinal oligometastatic disease.

\begin{tabular}{|l|l|l|l|l|l|}
\hline Study & Local Control Rate (\%) & \multicolumn{4}{l|}{ Months after SSRS treatment } \\
\hline & $\mathbf{0}$ & $\mathbf{1 2}$ & $\mathbf{1 4}$ & $\mathbf{2 4}$ & $\mathbf{6 0}$ \\
\hline Ho et al. 2016 & 100 & 85 & NR & $\mathbf{8 2}$ & $\mathbf{7 8}$ \\
\hline Chang et al. 2017 & 100 & 92 & NR & $\mathbf{8 6}$ & NR \\
\hline Chaw 2017 & 100 & NR & $\mathbf{8 3}$ & NR & NR \\
\hline Thibault et al. 2014 & 100 & 83 & NR & NR & NR \\
\hline Silva et al. 2018 & 100 & 100 & NR & NR & NR \\
\hline Barzilai et al. 2019 & 100 & 85 & NR & NR & NR \\
\hline Yamada et al. 2008 & 100 & NR & NR & 93 & NR \\
\hline Ahmed et al. 2012 & 100 & 88 & NR & NR & NR \\
\hline Kam et al. 2019 & 100 & 91 & NR & NR & NR \\
\hline Ave. & 100 & 89 & 83 & 87 & 78 \\
\hline Maximum & 0 & 11 & 0 & 6 & 0 \\
\hline Minimum & 0 & 6 & 0 & 5 & 0 \\
\hline
\end{tabular}

Abbreviations: NR - Not recorded, SSRS - Spinal Stereotactic Radiosurgery

Table III: Overall Survival rates of Radiosurgery Treatment of spinal oligometastatic disease.

\begin{tabular}{|c|c|c|c|c|c|}
\hline \multirow[t]{2}{*}{ Study } & \multirow{2}{*}{$\begin{array}{l}\text { Local Control } \\
\text { Rate }(\%)\end{array}$} & \multicolumn{4}{|c|}{$\begin{array}{l}\text { Months after Stereotactic } \\
\text { Radiosurgery treatment }\end{array}$} \\
\hline & & 12 & 14 & 24 & 60 \\
\hline Ho et al. 2016 & 100 & 95 & NR & 84 & 60 \\
\hline Chang et al. 2017 & 100 & 90 & NR & 76 & NR \\
\hline Chaw 2017 & 100 & NR & 95.7 & NR & NR \\
\hline Thibault et al. 2014 & 100 & 64 & NR & NR & NR \\
\hline Silva et al. 2018 & 100 & NR & NR & NR & NR \\
\hline Barzilai et al. 2019 & 100 & NR & NR & NR & NR \\
\hline Yamada et al. 2008 & 100 & NR & NR & NR & NR \\
\hline Ahmed et al. 2012 & 100 & 52 & NR & NR & NR \\
\hline Kam et al. 2019 & 100 & 80 & NR & NR & NR \\
\hline Ave. & 100 & 76 & 96 & 80 & 60 \\
\hline Maximum & $\mathbf{0}$ & 9 & $\mathbf{0}$ & 4 & $\mathbf{0}$ \\
\hline Minimum & $\mathbf{0}$ & 24 & $\mathbf{0}$ & 4 & $\mathbf{0}$ \\
\hline
\end{tabular}

Relationship between months after treatment with Stereotactic Radiosurgery against local control rate

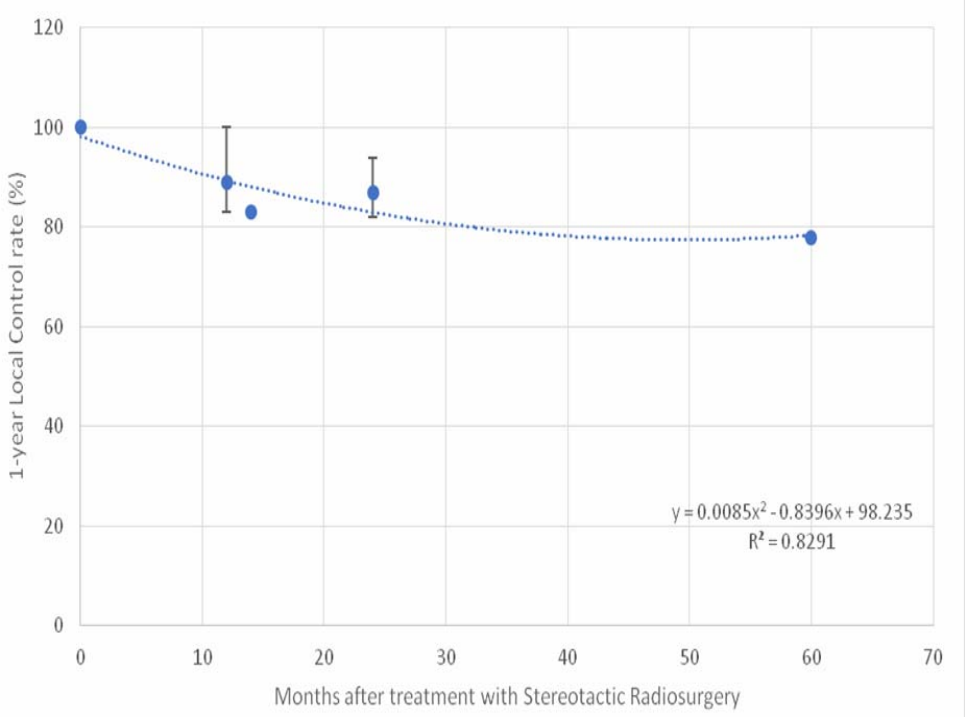

Graph 1: Relationship between months after treatment with Stereotactic Radiosurgery of spinal oligometastatic disease against average Local Control rate of included studies 


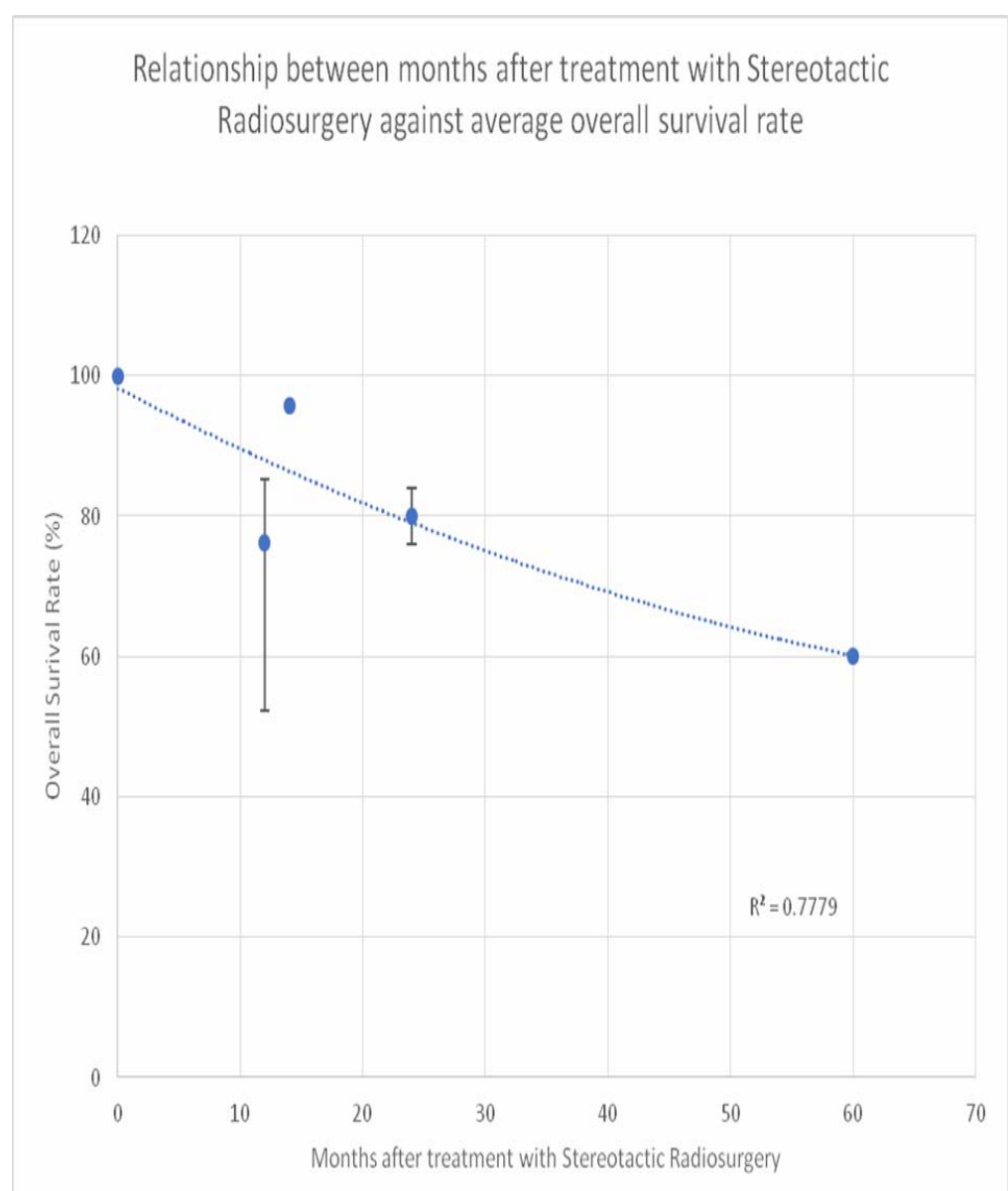

Graph 2: Relationship between months after treatment with Stereotactic Radiosurgery of spinal oligometastatic disease against average Overall Survival rate of included studies

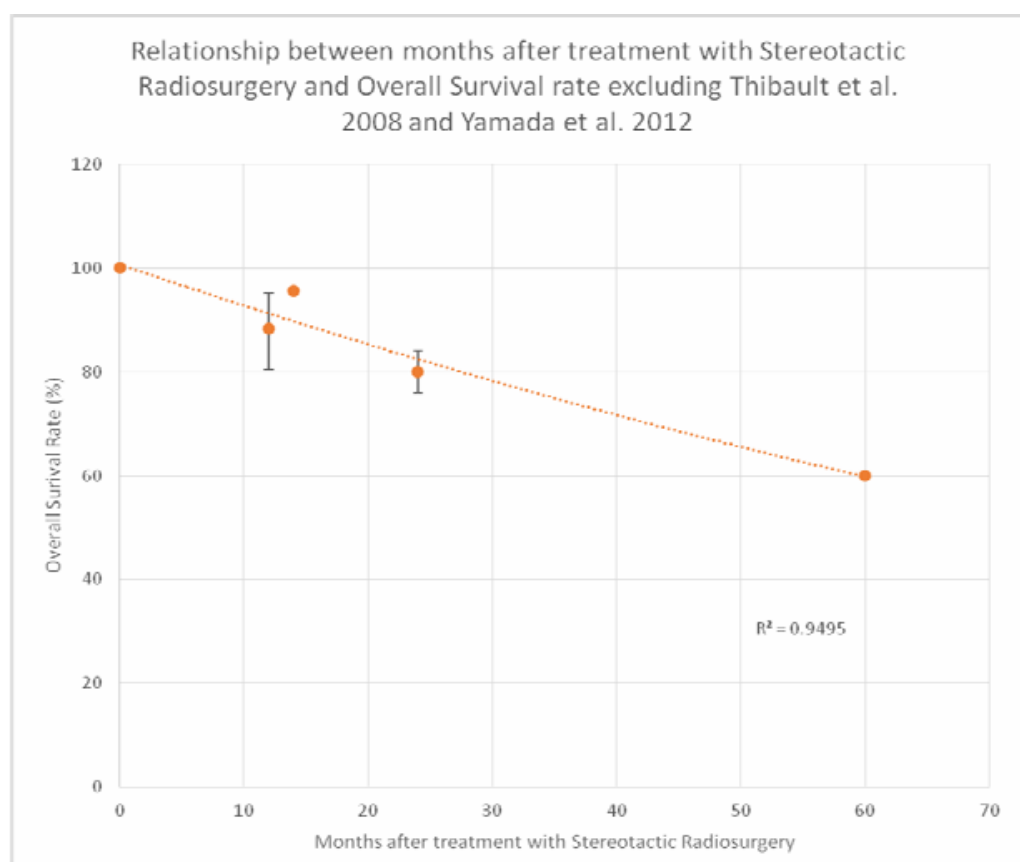

Graph 3: Relationship between months after treatment with Stereotactic Radiosurgery of spinal oligometastatic disease against average Overall Survival rate excluding Thibault et al. 2008 and Ahmed et al 2012. 


\section{Discussion}

This systematic review investigates the local control, overall survival and pain response of retrospective and prospective studies on the treatment of oligometastatic disease of the spine. When comparing local control rates, mostly a 1-year local control rate was given for most of the studies, Ho et al. is the only study out of the ones used in this systematic review that conducted a long term study with a follow up period up to 5 years which is unique and provides further evidence of local control and overall survival rates of stereotactic radiosurgery treatment. Barzilai et al. is also a key study because it provides evidence of SRS being a better modality of treatment versus surgery and cEBRT. All studies have been chosen given that the results they yielded were statistically significant $(\mathrm{P} \leq 0.05)$.

In graph 1 , a negative relationship between months after stereotactic radiosurgery and local control rate was established. It is important to note that none of the studies reporting results that were completely common with the other studies. Ho et al, provided the maximum amount of data and was the only study that reported local control at 60 months. Furthermore, standard deviation was not possible to be calculated for any of the data points except 12 months local control as 5 sets of data were not reported. Hence the use of minimum and maximum was utilized on data points that showed a range i.e. 12 months and 24 months. It is important to note that Silva et al. recorded $100 \%$ local control rate at 1 year which suggested that the all 25 patients didn't show signs of local failure after 1 year. This could either suggest very promising results of the treatment or incorrect collection of data at the follow up or perhaps the oligometastasis state was incorrectly diagnosed, as all the other studies suggest that there was local control was less than $100 \%$ at around the 1-year mark (Chaw 2017 recorded 14 months follow up local control). The average local control of the 9 studies was $89 \%$ (including Silva et al) at 1 year and $87 \%$ (without Silva et al), both figures suggesting excellent local control rates. Both figures are greater than Barzilai et al.'s local control rates of surgery with or without RT $(n=129)$ of $65.5 \%$ and cEBRT $(n=23)$ of $57.1 \%$. A polynomial trendline was drawn in graph 1 and derived $\mathrm{R}^{2}=0.8291$, which suggests that months after treatment and local control were reasonably negatively correlated. The plateau of the graph could also suggest that local control is not expected to deteriorate much after 60 months, however more longitudinal studies recording longer time periods needs to be investigated to confirm this.

Overall Survival was recorded in 6 of the 9 included studies, a negative relationship between months after SRS treatment and Overall Survival rate was established. Ho et al, Chang et al and Kam et al suggest overall survival rates above $80 \%$. Yamada et al and Ahmed et al derived quite low overall survival rates of $64 \%$ and $52 \%$ respectively. An average of $76 \%$ of 1 -year overall survival rate was calculated with inclusion of all 5 studies and $88 \%$ when excluding Yamada et al and Ahmed et al. A justification to exclude the two studies is that were conducted in 2008 and 2012 respectively, hence treatment method could be outdated as SRS technology may have drastically developed between 2012 and 2014. Hence graph 3 shows overall survival rate relationship excluding these two outliers and as a result it improves overall 1-year overall survival rate and error bar propagation, but also $\mathrm{R}^{2}=$ 0.9495 as opposed to $\mathrm{R}^{2}=0.7779$ in graph 2, suggesting a stronger relationship.

Chaw 2017 reported $95.7 \%$ overall survival at 14 months $(n=45)$ which is exceptionally high and lies above the trendline expectation in graph 2 and 
graph 3 but is included in data as there were no other studies that reported findings at 14 months and an average couldn't be calculated. There is a lack of data in this systematic review to make valid conclusions about overall survival rate however, it does provide a good indication that overall survival rates are excellent after 1 year.

Five studies reported pain response, 4 studies suggesting partial to complete pain relief, however Ahmed et al reporting increased pain after SRS was administered. Kat et al reported $66 \%$ of patients use of analgesics was absent at the 1 year follow up. Barzilai et al, quantified pain response with a pain numeric rating scale and reported drastic pain relief over 52 weeks. $80 \%$ of the studies that reported pain response provide improvement in pain suggesting SRS provides good to complete pain relief.

Investigating 601 patients, 70 patients (12\%) experienced Grade $1 / 2$ acute toxicities such as nausea, fatigue, pain flare, esophagitis, transient radiculitis, diarrhea, mucosal, skin, dysphagia. 5 $(<1 \%)$ patients experienced Grade 3 acute/late toxicities such as myelopathy and esophagitis. Twenty three (4\%) patients experiences vertebral compression fractures. This suggests that SSRS has excellent low toxicity levels in comparison to surgical interventions such en bloc spondylectomy.

Conclusion; Although this systematic review has indicated evidence for excellent local control, overall survival, pain response and toxicities for SSRS of spinal oligometastasis, it is imperative to conduct more investigations to increase investigated population samples and increase the validity of this review. More studies specifically of spinal oligometastasis should investigate surgical treatment such as separation surgery/en-bloc resection of need to be conducted in order to make a valid comparison as the study used in this review (Barzilai et. al) is insufficient. A univariate analysis of dosage fractionation could also be conducted to investigate the optimal dosage to ablate the tumor and control toxicities/pain ${ }^{19}$.

It is difficult to conclude results statistically, but it was attempted to plot the outcomes of radiosurgery graphically. However, to improve this review drastically, it is required to be compared to an abundant amount studies investigating local control and overall survival rates of surgical treatment and conventional external beam radiotherapy. However due to the limitations such as inadequate research on alternative treatments of spinal oligometastasis, it was not included in this review but was briefly mentioned in reference to a few studies. It is understandable there isn't much research on alternative treatments as it has been indicated by various studies mentioned in the review that radiosurgery is the optimal form of treatment for this form of cancer and could be unethical to provide treatment that has worse outcomes for patients. This review does conclude that radiosurgery is a promising modality of treatment for spinal oligometastatic disease as it increases survivability with low morbidity and toxicity. 


\section{References}

1. Hellman S, Weichselbaum R. Oligometastases. Journal of Clinical Oncology. 1995;13(1):8-10.

2. Belkacemi Y, Penel N. OLIGOMETASTATIC DISEASE A true clinical entity in Oncology?. [Internet]. Oncologypro.esmo.org. 2018 [cited 5 April 2020]. Available from: https://oncologypro.esmo.org/content/download/169948/3091363/file/ESMO-Clinical-Trials-Tipsand-Tricks-Oligometastatic-Disease.pdf

3. Zeng K, Tseng C, Soliman H, Weiss Y, Sahgal A, Myrehaug S. Stereotactic Body Radiotherapy (SBRT) for Oligometastatic Spine Metastases: An Overview. Frontiers in Oncology. 2019;9.

4. Laufer I, Rubin D, Lis E, Cox B, Stubblefield M, Yamada Y et al. The NOMS Framework: Approach to the Treatment of Spinal Metastatic Tumors. The Oncologist. 2013;18(6):744-751.

5. Tseng C, Eppinga W, Charest-Morin R, Soliman H, Myrehaug S, Maralani P et al. Spine Stereotactic Body Radiotherapy: Indications, Outcomes, and Points of Caution. Global Spine Journal. 2017;7(2):179-197.

6. Gerszten P. Spine Metastases: From Radiotherapy, Surgery, to Radiosurgery. Neurosurgery. 2014;61(CN_suppl_1):16-25.

7. Ho J, Tang C, Deegan B, Allen P, Jonasch E, Amini B et al. The use of spine stereotactic radiosurgery for oligometastatic disease. Journal of Neurosurgery: Spine. 2016;25(2):239-247.

8. Patchell R, Tibbs P, Regine W, Payne R, Saris S, Kryscio R et al. Direct decompressive surgical resection in the treatment of spinal cord compression caused by metastatic cancer: a randomised trial. The Lancet. 2005;366(9486):643-648.

9. Barzilai O, Versteeg A, Sahgal A, Rhines L, Bilsky M, Sciubba D et al. Survival, local control, and health-related quality of life in patients with oligometastatic and polymetastatic spinal tumors: A multicenter, international study. Cancer. 2018;125(5):770-778.

10. Stereotactic radiosurgery - Mayo Clinic [Internet]. Mayoclinic.org. 2020 [cited 5 April 2020]. Available from: https://www.mayoclinic.org/tests-procedures/stereotactic-radiosurgery/about/pac20384526

11. Moher D, Liberati A, Tetzlaff J, Altman D. Preferred reporting items for systematic reviews and meta-analyses: the PRISMA statement. BMJ. 2009;339(jul21 1):b2535-b2535.

12. Chang J, Gandhidasan S, Finnigan R, Whalley D, Nair R, Herschtal A et al. Stereotactic Ablative Body Radiotherapy for the Treatment of Spinal Oligometastases. Clinical Oncology. 2017;29(7):e119-e125.

13. Chaw C. Stereotactic Body Radiation Therapy for Oligometastatic Spinal Disease. International Journal of Radiation Oncology*Biology*Physics. 2017;99(2):E67.

14. Thibault I, Al-Omair A, Masucci G, Masson-Côté L, Lochray F, Korol R et al. Spine stereotactic body radiotherapy for renal cell cancer spinal metastases: analysis of outcomes and risk of vertebral compression fracture. Journal of Neurosurgery: Spine. 2014;21(5):711-718.

15. Silva S, Gliniewicz A, Martin B, Prabhu V, Germanwala A, Melian E et al. Oligometastatic Disease State Is Associated with Improved Local Control in Patients Undergoing Three or Five Fraction Spine Stereotactic Body Radiotherapy. World Neurosurgery. 2019;122:e342-e348.

16. Yamada Y, Bilsky M, Lovelock D, Venkatraman E, Toner S, Johnson J et al. High-Dose, SingleFraction Image-Guided Intensity-Modulated Radiotherapy for Metastatic Spinal Lesions. International Journal of Radiation Oncology*Biology*Physics. 2008;71(2):484-490.

17. Ahmed K, Stauder M, Miller R, Bauer H, Rose P, Olivier K et al. Stereotactic Body Radiation Therapy in Spinal Metastases. International Journal of Radiation Oncology*Biology*Physics. 2012;82(5):e803-e809.

18. Kam T, Chan O, Hung A, Yeung R. Utilization of stereotactic ablative radiotherapy in oligometastatic \& oligoprogressive skeletal metastases: Results and pattern of failure. Asia-Pacific Journal of Clinical Oncology. 2019;15(S2):14-19.

19. Dunne E, Fraser I, Liu M. Stereotactic body radiation therapy for lung, spine and oligometastatic disease: current evidence and future directions. Annals of Translational Medicine. 2018;6(14):283283. 